\title{
The role of trust in OSS communities - Case Linux Kernel community
}

\author{
Maria Antikainen ${ }^{1}$, Timo Aaltonen ${ }^{2}$, and Jaani Väisänen ${ }^{3}$ \\ 1 VTT Technical Research Centre of Finland, Media and Mobile \\ Usability, Sinitaival 6, 33101Tampere, Finland \\ maria.antikainen@vtt.fi \\ 2 Tampere University of Technology, Institute of Software Systems, \\ Korkeakoulunkatu 1, 33720 Tampere, Finland \\ timo.aaltonen@tut.fi \\ 3 Tampere University of Technology, Institute of Business Information \\ Management, Korkeakoulunkatu 8, 33720 Tampere, Finland \\ jaani.vaisanen@tut.fi
}

\begin{abstract}
Open source software development has been the subject of interest among businesses as well as in the academic world. OSS enables many possibilities for companies but also sets new kinds of challenges. Because of the characteristics of the OSS phenomenon we propose that trust in OSS communities plays a key role in facilitating their success. Therefore, the aim of this study is to explore the factors that affect trust in OSS communities. The data is gathered by a survey aimed to Linux Kernel developers. Among other results it may be concluded that the most important factors affecting trust seem to be other developers' skills, reputation as well as the formal and informal practices.
\end{abstract}

Keywords: OSS community, Linux Kernel, trust, case study, survey

\section{Introduction}

Open source software development has been the subject of interest among businesses as well as in the academic world. OSS enables many possibilities for companies but also sets new kinds of challenges. In this study we propose that trust in OSS communities plays a key role in facilitating their success. Trust is important in this context because the participants of OSS communities represent different organizations and different motivations, and therefore, there is constant risk for opportunistic behavior between participants. Trust is also a central factor when organizations are making decisions about whether they choose OS software or not.

Please use the following format when citing this chapter:

Antikainen, M., Aaltonen, T. and Väisänen, J., 2007, in IFIP International Federation for Information Processing, Volume 234, Open Source Development, Adoption and Innovation, eds. J. Feller, Fitzgerald, B., Scacchi, W., Sillitti, A., (Boston: Springer), pp. 223-228. 
Therefore, the aim of this study is to explore the factors that affect trust in OSS communities. Since the study area is rather unexplored, the study starts with scrutinizing deeply one case that is the Linux Kernel community. The actual study consists of two phases, starting with exploring the trust in OSS communities with the multidisciplinary literature analysis, a phase which is reported in Antikainen and Aaltonen [1]. As a result of the first phase, the preliminary model of trust in the Linux Kernel community was built. In this paper we focus on a web-based survey aimed for the Linux Kernel developers to be able to test our preliminary model of trust.

In this paper we define trust as "the extent to which a person is confident in and willing to act on the basis of, the words, actions, and decisions of another" [2]. Trust occurs in a relationship between a trustor and trust target. Fundamentally, we see that trust implies a party's willingness to accept vulnerability but with an expectation or confidence that it can rely on the other party [3], [4], [5].

\section{Linux Kernel community}

Nowadays Linux has grown to the largest open source project in the world. [6] Linus Torvalds is still the benevolent dictator of Linux as he is the final arbiter of all changes accepted into the Linux kernel. An often-referred model of an open source community is presented in Nakakoji et al. [7]. The model describes an open source community as an onion, where the most influential roles are in the center, and each outer layer consists of less and less influential ones - see the left-hand side of Figure 1. The right-hand side of the figure depicts the onion-like figure instantiated to the Linux Kernel community.
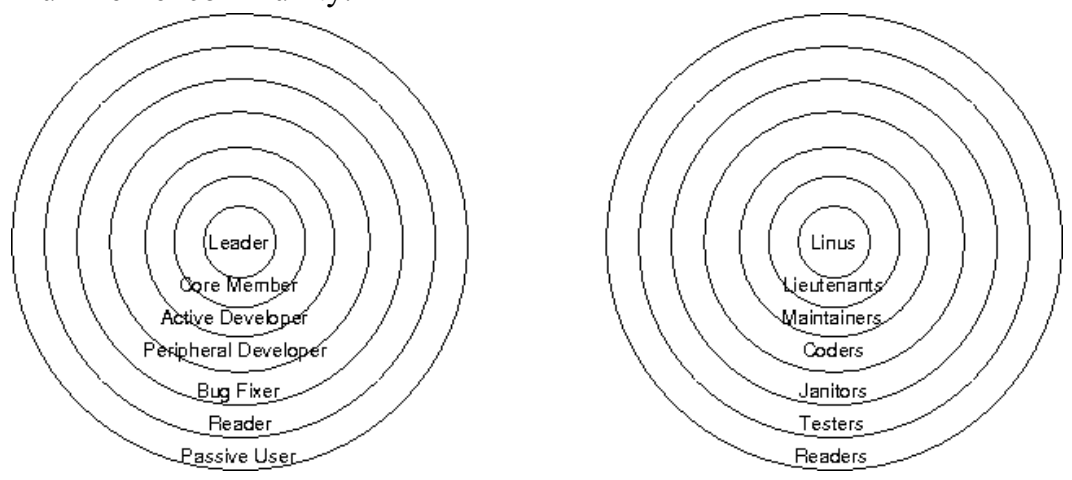

Fig. 1. The general onion model of open source communities vs. the Linux Kernel onion model

Developing open-source software is based on making small changes to an existing code. In Linux a description of such a change is called a patch. Certain conventions 
are followed when a programmer attempts to get her patch "upstream" (to get the patch to the Linus' kernel).[8].

There is no formal process for developing Linux [9]. The unplanned emergence of lieutenants and the convention that patches are delivered to them is de facto behaviour of the community which takes place in mailing lists. Thus, there exists an implicit "control" for organizing the community. People who are skilled and have a large impact in the development simply conceive more power.

Developers who become lieutenants are trusted by the programmers who start submitting their patches to them. Actually there exists a chain of trust in the community, since programmers trust lieutenants who in turn trust Linus. The chains of trust are made explicit by adding signoffs to the patches: the programmer signs the patch she made before delivery, and then if the patch is accepted by maintainer she also signs of the patch and so on. Each patch includes a chain of trust in an explicit form [10]. Precise rules for signing patches can be found at Open Source Development Labs, Inc [11].

\section{Methodology}

The study uses case study method exploring the Linux Kernel community. The community was chosen based on the structure and the sufficient size of the community, which enabled the collection of the needed amount of data. A web-based survey was created and linked to the Linux Kernel community's main mailing list. The research instrument was administered via a web-based survey tool provider, SurveyMonkey (www.surveymonkey.com). The questions were based on the preliminary framework created in the phase 1. Yet, the questions are not restricted to this framework so that exploratory approach to the phenomenon could be applied.

The study resulted with 139 answers during 12 days. However, since the web questionnaire was divided into several sections, the amount of respondents decreased towards the end of the questionnaire to 95 respondents. Also open questions got fewer responses since they were not obligatory; however, the information from open questions was very valuable.

Analysis of variance was used to gain understanding about the differences between the different factors of trust. Cronbach's Alpha $(0,759)$ was measured to validate the reliability of the questionnaire as well.

\section{Factors affecting trust}

The survey included questions considering the background of the developers, the nature of their participation in Linux Kernel community as well as questions considering trust. The factors of the preliminary model of trust were asked with multiple questions. In the following the results are briefly discussed and illustrated. 
To be able to test the preliminary model and find out the importance of different factors we constructed one common index that is comparable throughout all the factors by calculating the mean results of each factor. The significance of the differences in means was concluded by analysis of variance. There has been discussion regarding the use of ANOVA when dealing with ordinal and grouped variables. Although the formulae behind ANOVA require computations that would normally need scale variables, there is a predominant conception that has been reviewed e.g. in Brockett \& Golden [12] that states that the error margin from using interval level methods for ordinal data is very small. Therefore, the authors have decided that the use of ANOVA is acceptable in this situation. The mean values are illustrated in Figure 2.

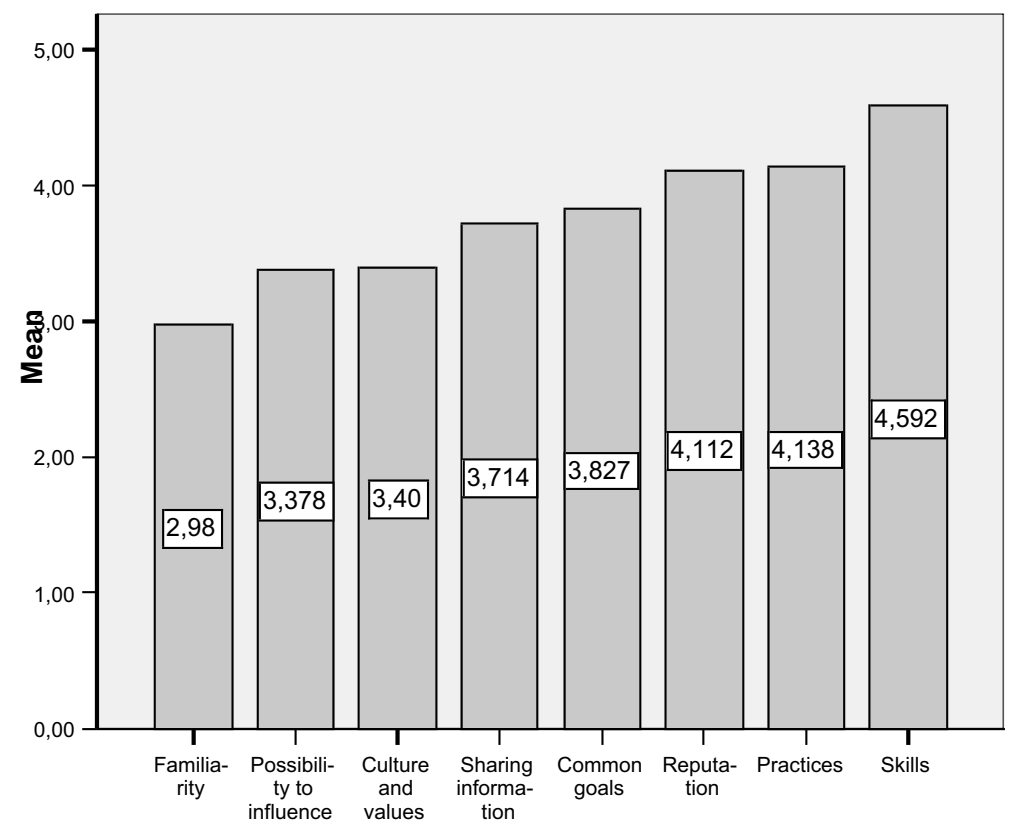

Fig. 2. The means of the factors of trust

Based on the results, 'skills' factor seems to be most important one followed by 'practices' and 'reputation' with almost similar means. Next comes 'common goals' and 'sharing information'. The least important factors seem to be 'the culture and values', 'the possibility to influence' and 'familiarity'. Analysis of variance showed that differences between sharing information and the common goals were not significant. Also differences between 'possibility to influence' and 'culture and values' can derive from coincidence. Again 'reputation' and 'practices' do not have significant differences in their means. Since the values were quite high, based on the analyses conducted, none of the chosen factors can be removed from the model. 
The final question considered how the respondents see the role of trust in the Linux Kernel community. We got altogether 49 answers to this open question of which 4 were discarded. More than a half of the answers included the statement ' $y e s$ ' (26), especially general yes was popular. Some of the respondents who answered yes specified their answers adding that they trusted towards the core of the onion (see Figure 1), or towards outer layers of onion. Trust towards the core of the onion was considered less important, and trust towards outer layers was mentioned only couple of answers. In contrast, we got also an amount of plain no answers (3).

Other answers considered the trust issue in general level without commenting directly if they trust into Linux Kernel community or not. We divided these answers into three categories: trust in code (11), trust in process (3), and trust based on reputation (4). However, it can be argued that trust in process and trust in code may seem to belong to the same category. Then one third of the respondents would have chosen this compound category. This open question seems to affirm the results (Fig.2) that skills, practices and reputation have an important role in trust towards Linux Kernel community.

\section{Conclusions}

In this paper we studied factors influencing trust in the Linux Kernel community. Based on the results we propose that eight factors presented in our model seem to affect trust in the Linux Kernel community. The most significant factors seem to be trust towards other developers' skills, their reputation and informal and formal practices in the community. Also the answers for open question confirmed this conclusion. Due to the fact that Linux Kernel is one of the most complex open source products available, skills and reputation of other members of the community are naturally appreciated. Explanation for the value of practices may be that the conventional software engineering methods are substituted by informal practices. Without them the community would not function.

The least significant factors are familiarity, the possibility to influence and the culture and values. The Linux Kernel community is extremely large, and people are not very familiar with each other. Therefore, familiarity cannot be ranked high. Linux - like most open source projects - is based on a benevolent dictatorship. This fact and the size of the community set limits to possibility to influence. Maybe people willing to gain much influence do not join this kind of communities. Linus Torvalds is a very pragmatic person and he makes sure that Linux is not considered as free software but open source and in such atmosphere the culture and values cannot be significant factors. Therefore, applying this survey to smaller communities might lead to different results in this factor.

This study contributes to a clarification of the role of trust in a one type of OSS community. By testing the preliminary model of trust and measuring the importance of different factors, this study brings valuable knowledge on the dynamics of OSS communities. The present study represents an opening for further studies concerning 
the role of trust in different kinds on OSS communities aimed for building a generalized model of trust in such communities. As mentioned before, surveying several communities might lead to interesting differences between them.

\section{References}

[1] Antikainen, M. and Aaltonen, T. (2007). In Helander, N. \& Antikainen, M. (eds.), Essays on OSS practices and sustainability. e-Business Research Center Research Reports 36. Tampere University of Technology \& University of Technology.

[2] McAllister, D. 1995. Affect- and cognition-based trust as foundations for interpersonal cooperation in organizations. Academy of Management Journal, 38 (1), 24-59.

[3] Lewicki, R., McAllister, D. and Bies, R. (1998). Trust and distrust: New relationships and realities. The Academy of Management Review, 23 (3), 438-458.

[4] Moorman, C., Zaltman, G. and Deshpandè, R. (1992). Relationships between providers and users of market research: The dynamics of trust within and between organizations. Journal of Marketing Research, 29(3), 314-328.

[5] Morgan R. M. and Hunt S.D. (1994). The commitment-trust theory of relationship marketing. Journal of Marketing, 58 (July), 20-38.

[6] The Penguin's Window: Corporate Brands From an Open-Source Perspective. Journal of the Academy of Marketing Science, Vol. 34, No. 2, 115-127 (2006).

[7] Nakakoji, K., Yamamoto, Y., Nishinaka, Y., Kishida, K. and Ye, Y. (2002). Evolution patterns of open-source software systems and communities. Proceedings of International Workshop on Principles of Software Evolution.

[8] Site kernelnewbies.org on the www.

[9] Linus Torvalds' Benevolent Dictatorship. BusinessWeek magazine August 182004.

[10] Andrews J. (2004). Linux: Documenting How Patches Reach The Kernel. URL http://kerneltrap.org/node/3180 on the computing news website Kerneltrap.

[11] Open Source Development Labs, Inc. (2005). Developer's Certificate of Origin 1.1. At www page http://www.osdl.org/newsroom/press_releases/2004/2004_05_24_dco.html.

[12] Brockett, P. and Golden, L. (1992). A Comment on "Using Rank Values as an Interval Scale" by Dowling and Midgley. Psychology and Marketing, 9(3), 255-261. 